\title{
Bestimmungsgründe der öffentlichen Kleinkinderbetreuung im regionalen Vergleich
}

Im August 2013 soll ein bundesweiter Rechtsanspruch auf öffentliche Betreuung für Kinder unter drei Jahren in Kraft treten. Allerdings bestehen erhebliche regionale Unterschiede beim Ausbau der Kleinkinderbetreuung. Während sich in Amberg nur 10,5 \% der Kleinkinder in öffentlicher Betreuung befinden, sind es im Jerichower Land 63,3\%. Der Beitrag analysiert die sozioökonomischen, institutionellen und politischen Ursachen der regionalen Unterschiede.

ERIC SEILS, DANIEL MEYER

\section{Einleitung}

Der Ausbau der öffentlichen Kinderbetreuung für unter 3-Jährige stellt derzeit eine zentrale Herausforderung der Familienpolitik dar. Im Jahre 2008 wurde das Kinderförderungsgesetz (KiföG) mit den Stimmen von Union und SPD verabschiedet. Dieses schreibt unter anderem fest, dass ab August 2013 alle Kleinkinder ab Vollendung des ersten Lebensjahres einen Rechtsanspruch auf einen Betreuungsplatz haben. Allerdings ist fraglich, ob die Betreuungsgarantie wirklich umgesetzt werden kann. Den Angaben des Statistischen Bundesamtes zufolge fehlten im März 2012 noch 220.000 Plätze für eine ausreichende Versorgung (Statistisches Bundesamt 2012a; 2012b, S. 7). Sollte es bis zum angegebenen Stichtag nicht gelingen, ausreichend Betreuungsplätze zur Verfügung zu stellen, droht den Kommunen eine Klagewelle vonseiten derjenigen Eltern, welche für ihren Nachwuchs keinen Platz finden konnten. Aktuelle Daten der Statistischen Ämter des Bundes und der Länder weisen zudem darauf hin, dass die Betreuungssituation auch 2012 trotz bundesgesetzlicher Betreuungsgarantie weiterhin stark regional variiert (Statistische Ämter des Bundes und der Länder 2012, S. 9).

Der nachfolgende Beitrag beschäftigt sich mit der Frage, welche Faktoren für die regionalen Unterschiede verantwortlich sind. Die international vergleichende Wohlfahrtsstaatsforschung hat sich im letzten Jahrzehnt zunehmend den Ursachen für die Unterschiede bei den sozialen Dienst- leistungen zugewandt (Bonoli/Palier 2007; Bonoli/Reber 2010; Fargion 2002; Huber/Stephens 2000; Randall 2000). Zur Erklärung wird vornehmlich auf drei klassische Theorien der Staatstätigkeitsforschung, namentlich den Funktionalismus, die Parteiendifferenztheorie und den Institutionalismus zurückgegriffen. Diese haben bei der Erklärung des Aufbaus von Transferprogrammen gute Dienste geleistet. Allerdings haben sich die Rahmenbedingungen gewandelt, und es muss sich noch zeigen, inwiefern sie auf soziale Dienstleistungen in einer Zeit knapper öffentlicher Haushalte angewandt werden können.

Der folgende Abschnitt befasst sich mit der Frage, inwiefern die Theorien der internationalen Staatstätigkeitsforschung für das junge Forschungsfeld zur Kleinkinderbetreuung auf regionaler Ebene adaptiert und entsprechende Hypothesen generiert werden können. Anschließend wird auf die Datenbasis und das methodische Vorgehen eingegangen. Im vierten Abschnitt werden die Hypothesen empirisch getestet. Abschließend werden die Ergebnisse knapp zusammengefasst.

\section{Theorien der Staatstätigkeit}

Die vergleichende Wohlfahrtsstaatsforschung hat bei der Erklärung von Unterschieden sozialstaatlicher Ent- 
wicklung in den letzten Jahrzehnten häufig auf die Theorien der Staatstätigkeitsforschung zurückgegriffen. Dies sind der Funktionalismus, die Parteiendifferenztheorie, der Institutionalismus und der Machtressourcenansatz. Letzterer kann auf regionaler Ebene jedoch nicht getestet werden, da keine Daten zur Stärke der Gewerkschaften nach Kreisen vorliegen. Im Folgenden wird daher nur auf die Literatur zum Funktionalismus, der Parteiendifferenztheorie und zum Institutionalismus eingegangen. Dabei wird geklärt, inwiefern aus den internationalen Theorien der Staatstätigkeit Hypothesen abgeleitet werden können, die geeignet sind, regionale Unterschiede beim Ausbau der öffentlichen Kinderbetreuung zu erklären.

\subsection{Funktionalismus}

Die bis in die 1970er Jahre dominierenden Funktionalisten sahen in Sozialstaaten die notwendige Konsequenz der Industrialisierung. Der wirtschaftliche Wandel sorgte demnach dafür, dass Funktionen, die einst in der Familie oder im Dorf erbracht, nun in den Bereich der Geldwirtschaft übertragen wurden. Dieser Vorgang war einerseits mit einem Zuwachs an materiellen Ressourcen verbunden, bewirkte aber andererseits auch einen Verlust traditioneller Arbeitsformen und Familienstrukturen. Im Kontext der Industrialisierung meinte dies nicht nur neue Arbeitsinhalte, sondern auch eine zunehmende Labilität der neuen Beschäftigungsverhältnisse sowie den Übergang von der Groß- zur Kleinfamilie (Achinger 1979 [1958], S. 18ff.). Sozialstaaten greifen auf die neu geschaffenen Ressourcen zurück, um dem Erfordernis neuer gesellschaftlicher Formen zu entsprechen (ebenda). Die Logik der Industrialisierung führt also unabhängig vom politischen System und der parteipolitischen Ausrichtung der Regierung zu einem Niveau an Sozialleistungen, welches durch den wirtschaftlichen Entwicklungsstand geprägt ist (Wilensky 1975; Zöllner 1963, S. 27ff.).

Wenngleich der Funktionalismus im engeren Sinne seit Ende der 1970er Jahre weithin in Ungnade gefallen ist, wird auch heute noch das Für und Wider von Sozialleistungen mit gesellschaftlichen und ökonomischen Erfordernissen begründet. In der Tat bringt auch der Trend zur Dienstleistungsgesellschaft neue gesellschaftliche Ressourcen und Erfordernisse mit sich. Einen nicht unbedeutenden Teil der neuen Dienstleistungen machen Tätigkeiten aus, die in der Vergangenheit im Rahmen der unbezahlten Hauswirtschaft erbracht wurden (Esping-Andersen 1999). Aus dieser Perspektive stellt die Auslagerung der Kindererziehung aus der Familie auf öffentliche oder private Betreuungseinrichtungen einen weiteren Schritt in der Monetarisierung der Hauswirtschaft dar. Die dadurch ermöglichte Arbeitsmarktpartizipation von Frauen kann als neue Erwerbsform interpretiert werden. Die mit der Frauenerwerbstätigkeit einhergehenden Effizienzgewinne schaffen zusätzlichen Wohlstand. Wie einst die Industrialisierung (Achinger 1979 [1958], S. 27ff.) geht auch die Deindustrialisierung mit neuen Familienstrukturen einher. Dieser Wandel erfordert eine neue Sozialpolitik. So knüpfte die Sozialpolitik der Nachkriegszeit an der Kleinfamilie und der lebenslangen Ehe an. Frau und Kinder erhielten dabei abgeleitete Leistungen aus der Erwerbstätigkeit des Mannes. In den letzten Jahrzehnten wurden die Ehen jedoch zunehmend brüchiger. In der Folge hat die Zahl der Haushalte von Alleinerziehenden zugenommen, die besonders häufig von Armut bedroht sind. Der Ausbau der Kinderbetreuung soll daher die Erwerbstätigkeit von (alleinstehenden) Müttern unterstützen, um Kinderarmut zu vermeiden (Esping-Andersen 2002; Jaumotte 2003; OECD 2011; Whiteford/Adema 2007).

Daraus ergeben sich folgende Hypothesen: Ein hoher wirtschaftlicher Entwicklungsstand und eine verbreitete Frauenerwerbstätigkeit lassen auf eine ausgebaute öffentliche Betreuungsinfrastruktur schließen. Außerdem sollte die Kinderbetreuung dort ausgebaut sein, wo Ehescheidungen und Kinderarmut verbreitet sind.

\subsection{Parteiendifferenztheorie}

Seit der zweiten Hälfte der 1970er Jahre fand die Rolle der parteipolitischen Zusammensetzung der Regierung in der Staatstätigkeitsforschung zunehmende Beachtung. Erstmals wurde dieser Gedanke von Douglas Hibbs (1977) formuliert. Für den hiesigen Kontext ist erstens entscheidend, dass sich die Interessen der Wählerschaft nach Hibbs' Modell allein aus ihren ökonomischen Interessen ergeben. Dementsprechend lassen sich Parteien und Wählerschaften im traditionellen Links-Rechts-Schema verorten (Hibbs 1977, S. 1470), welches sich am Klassenkonflikt orientiert. Zweitens verfolgen die Parteien, wenn sie an der Regierung sind, genau die Politik, welche ihrer Klientel dient. Das heißt, dass die Regierungsparteien die Politiken anbieten, welche ihre Klientel nachfragt.

Die Parteienforschung der letzten Jahrzehnte hat allerdings beide Punkte in Zweifel gezogen: Erstens ist inzwischen klar, dass sich die politischen Präferenzen von Individuen nicht allein aus deren Position im Klassenkonflikt ableiten lassen. So wurde früh erkannt, dass christdemokratische Parteien nicht einfach als „rechte“ Parteien zu betrachten sind, sondern vielmehr zum Aufbau der Sozialstaaten beigetragen haben (Huber et al. 1993; Wilensky 1981). Konnten die christdemokratischen Parteien noch als Ausnahmefall oder Kodierungsproblem angesehen werden, wurde mit dem Aufkommen der „neuen sozialen Bewegungen" in den 1980er Jahren deutlich, dass sich die Wählerlandschaft veränderte.

Herbert Kitschelt (1953) hat daher einen Versuch unternommen, den Raum politischer Präferenzen in einem Koordinatensystem neu zu fassen. Die horizontale Achse erstreckt sich von ökonomisch linken (socialist) zu ökonomisch rechten (capitalist) Positionen. Die vertikale Achse reicht von progressiven (libertarian) zu konservativen (authoritarian) Positionen. Entscheidend für die Position auf der Links-Rechts-Achse sind Markterfahrungen. Lebt eine Person von Arbeits- oder Kapitaleinkommen, arbeitet sie in 
der Binnenwirtschaft oder im internationalen Sektor? Die Position auf der Progressiv-Konservativ-Achse hängt hingegen von der Organisationserfahrung ab. Welches Geschlecht hat die Person? Wie gebildet ist eine Wählerin? Welche Arbeiten verrichtet sie? Darüber hinaus können auch Erfahrungen außerhalb der Arbeitswelt wie etwa die Arbeit in der Familie die politischen Präferenzen prägen. In diesem Koordinatensystem verteilen sich die Wähler hauptsächlich entlang einer Geraden von links-progressiven bis zu rechts-konservativen Positionen. Auch die Präferenzen bezüglich des Umgangs mit den „neuen sozialen Risiken“ wie der Kindererziehung sind durch ein solches ergänztes Schema geprägt (Kitschelt/Rehm 2006). Aus dieser Perspektive entwickeln vor allem junge, gebildete Frauen, die im öffentlichen Sektor arbeiten, eine Nachfrage links-progressiver Politiken wie etwa der Kinderbetreuung.

Wenn Parteien lediglich die politischen Präferenzen ihrer Klientel umsetzen würden, wäre im Kontext der Bundesrepublik zu erwarten, dass öffentliche Kinderbetreuung dort ausgebaut wird, wo die links-progressive Partei der GRÜNEN viele Wählerinnen hat. Von einer traditionell linken Wählerschaft ist hingegen ein geringeres Interesse am Ausbau der Kinderbetreuung zu erwarten, weil traditionelle sozialdemokratische Parteien zwar links, aber nicht sonderlich progressiv sind. Ein hoher Anteil von CDU/CSUWählern lässt hingegen erwarten, dass der Anteil öffentlich betreuter Kinder klein ist, weil die christliche Wählerschaft konservative Präferenzen hat und die Kinderbetreuung aus ihrer Sicht durch Frauen in der Familie erfolgen sollte (Henninger/von Wahl 2010).

Zweitens ist zu berücksichtigen, dass Kitschelt auch die These, wonach Regierungsparteien einfach die Interessen ihrer Klientel umsetzen, in Zweifel gezogen hat. Parteiführer sind ihm zufolge in der Lage, auf die sich verändernde Wählerlandschaft durch Veränderungen des Politikangebotes strategisch zu reagieren. Sie berücksichtigen nicht nur die Verteilung der Wählerschaft, sondern reagieren auch auf die Konkurrenz durch andere Parteien (Kitschelt 1993; 1999). Die Politik, welche eine Regierungspartei umsetzt, muss also nicht zwingend mit den politischen Präferenzen ihrer Klientel übereinstimmen.

\subsection{Institutionalismus}

Mitte der 1980er Jahre wurde die Forderung erhoben, die Rolle von Institutionen bei der Analyse sozialpolitischer Willensbildungsprozesse stärker zu berücksichtigen (Orloff/ Skocpol 1984; Pierson/Skocpol 2000). Autorinnen aus dieser Richtung betonen, dass Entscheidungen über Sozialstaatsreformen nicht am grünen Tisch, sondern in Abhängigkeit von bereits vorhandenen sozialstaatlichen Arrangements gefällt werden. Dieses ,institutionelle Erbe“ hat einen Einfluss auf die zukünftige Politik, indem es bestimmte Politiken nahe legt und andere mit hohen (politischen) Kosten belegt. In den letzten Jahrzehnten haben sich Institutionalisten vor allem dadurch hervorgetan, dass sie die Stabilität wohlfahrtsstaatlicher Institutionen im Kontext der Austeritätspolitik (Esping-Andersen 1996, S. 24) mit Mechanismen der Pfadabhängigkeit begründeten (Pierson 1994; 1996; 2000).

In der vergleichenden Literatur wird nun argumentiert, dass die Entwicklung der öffentlichen Kinderbetreuung vom Zeitpunkt abhängt, zu dem mit dem Aufbau der Kinderbetreuung begonnen wird (Bonoli 2007; Fargion 2002). Bei Ländern, die in der Prosperitätsphase vor der ersten Ölkrise mit dem Aufbau einer Betreuungsinfrastruktur begonnen haben, greifen danach die selbstverstärkenden Mechanismen der Pfadabhängigkeit (Ellingsæeter 2008; Fargion 2002, S. 67ff.). Für diese programmspezifische Pfadabhängigkeit lassen sich mindestens zwei Gründe anführen: Erstens verringert sich bei zunehmender öffentlicher Kinderbetreuung die Zahl der Spielkameraden, die ein von der Mutter betreutes Kind auf dem Spielplatz vorfindet. Befinden sich wie in Dänemark - fast alle Kinder in öffentlicher Betreuung, dann führen Kinder außerhalb der Kindergärten ein relativ einsames Leben (Emerek 2008, S. 11). Dieser soziale Mechanismus steigert somit die Nachfrage nach öffentlicher Betreuung. Zweitens verschieben sich mit dem Ausbau der Kinderbetreuung die Normen. Je mehr Eltern ihre Kinder in öffentliche Betreuung geben, desto eher wird dies zunächst gesellschaftlich akzeptiert, bis es schließlich zur Norm wird und Eltern dazu drängt, ihre Kinder in öffentliche Betreuung zu geben.

Länder, die erst in der Phase der Sparpolitik mit dem Aufbau der öffentlichen Kinderbetreuung beginnen, sehen sich dieser These zufolge unüberwindlichen Finanzierungsproblemen gegenübergestellt, welche vor allem aus den wachsenden Ausgaben für die Renten resultieren. Ausgaben für Alte verdrängen Ausgaben für den Aufbau einer öffentlichen Kinderbetreuung (crowding out). Die Folge ist, dass diese Länder über eine allenfalls schwache öffentliche Kinderbetreuung verfügen und diese trotz wachsenden Bedarfs auch nicht weiter ausbauen. Diese beiden Ländergruppen würden daher ihrem jeweils eigenen Pfad folgen, ohne dass es zu einer Konvergenz käme.

Die Kombination der Thesen von der programmspezifischen Pfadabhängigkeit und der crowding-out-These mit der Konsequenz dauerhafter Unterschiede bei der Kinderbetreuung wirft für die Analyse regionaler Varianz der Kinderbetreuung in der Bundesrepublik interessante Perspektiven auf. Erstens ist die vorliegende Studie nicht zuletzt durch das Fortbestehen großer regionaler Unterschiede trotz nationaler Gesetzgebung motiviert. Zweitens bestand in den fünf neuen Bundesländern bereits vor dem Beitritt zur Bundesrepublik eine ausgebaute Tagesbetreuung für Kleinkinder. In den alten Bundesländern war die Zahl der Plätze für Kleinkinder hingegen verschwindend gering. Zwar fallen Alterssicherungsausgaben bei den Kommunen nur in Form der Pensionen städtischer Beamter an, jedoch stehen sie unter einer recht strikten Budgetrestriktion, die sich aus den begrenzten Kompetenzen zur Abgabenerhebung ergibt. Die Thesen von Fargion und Bonoli haben für den 
regionalen Vergleich also die folgenden Implikationen: Erstens sollte die Betreuungsquote im Osten weiter steigen. Zweitens ist demnach zu erwarten, dass die Betreuungsquoten der Kleinkinder im Westen nicht steigen. Die Betreuungsquoten der Kleinkinder sollten also nicht konvergieren.

\section{Datenbasis und methodisches Vorgehen}

Um die aufgestellten Hypothesen empirisch überprüfen zu können, müssen die zentralen Konzepte zunächst durch geeignete Indikatoren operationalisiert werden. Zunächst zur abhängigen Variablen dieser Untersuchung: Der Ausbau der öffentlichen Kleinkinderbetreuung in den deutschen Kreisen und kreisfreien Städten wird mit Hilfe von Betreuungsquoten gemessen. Betreuungsquoten geben den Anteil der betreuten Kleinkinder unter drei Jahren in einer Kindertageseinrichtung oder in Kindertagespflege an allen Kindern dieser Altersgruppe an. Nun kann zu Recht eingewendet werden, dass Betreuungsquoten keine angemessene Kennziffer für den Ausbau der Kinderbetreuung darstellen, weil sie lediglich die Anzahl der tatsächlich betreuten Kinder und nicht die Anzahl der vorhandenen Plätze - wiedergeben. Es handelt sich also um einen Indikator, der weniger das Angebot als vielmehr die befriedigte Nachfrage misst. Andererseits sorgt die angespannte Betreuungssituation in dieser Altersgruppe zumindest im Westen dafür, dass das vorhandene Angebot weitgehend ausgeschöpft wird. Unter der Annahme, dass die Anzahl der betreuten Kinder durch das Angebot beschränkt, d.h. die Nachfrage nicht befriedigt ist, sind Betreuungsquoten eine geeignete Kennziffer, um die Ausdehnung der Betreuungsinfrastruktur für diese Altersgruppe zu messen. Ferner stellen Betreuungsquoten für das Bundesfamilienministerium den zentralen Erfolgsmaßstab bei der Umsetzung der beschlossenen Betreuungsgarantie dar. Bei den Kinderbetreuungsquoten handelt es sich um Stichtagsdaten auf der Ebene von Kreisen. Für diese Untersuchung werden die Daten von 2007 bis 2012 aus den (elektronischen) Anhangstabellen der Publikationen zur „Kindertagesbetreuung regional“ herangezogen (Statistische Ämter des Bundes und der Länder 2007 - 2012). In der multivariaten Querschnittsanalyse beziehen sich die Kinderbetreuungsquoten ausschließlich auf den aktuellen Rand (2012).

Die theoretisch interessierenden unabhängigen Variablen wurden grundsätzlich mit den neuesten verfügbaren Daten operationalisiert. In vielen Fällen muss jedoch auf Daten aus den vergangenen Jahren zurückgegriffen werden, da regional gegliederte Zahlen erst mit zeitlicher Verzögerung vorliegen. Für die Analyse erwächst hieraus kein Problem, weil die relativen Unterschiede zwischen den einzelnen Regionen im Verlauf weniger Jahre praktisch konstant bleiben.

Wie in der international vergleichenden Forschung üblich, wird der wirtschaftliche Entwicklungsstand einer Re- gion durch das Bruttoinlandsprodukt (BIP) je Einwohner gemessen. Die Frauenerwerbstätigkeit wird als Anteil sozialversicherungspflichtig beschäftigter Frauen an der weiblichen Bevölkerung im Alter von 15 bis unter 65 Jahren operationalisiert. Darüber hinaus wird der Anteil der in Teilzeit beschäftigten Frauen berücksichtigt, weil sich die Betreuungsgarantie nur auf einen Teilzeitplatz bezieht. Die gewandelten Familienstrukturen werden durch die Anzahl der Ehescheidungen je 1.000 Einwohner über 18 Jahren abgebildet. Die Statistik zu den Ehescheidungen im Jahr 2011 wurde freundlicherweise durch das Statistische Bundesamt zur Verfügung gestellt. Als Indikator für Kinderarmut wurden die SGB-II-Hilfequoten der unter 3-Jährigen als Anteil der Kleinkinder in Bedarfsgemeinschaften an allen unter 3-Jährigen erfasst.

Die Unterstützung einzelner Parteien in der Wählerschaft wird über den Stimmenanteil gemessen, den sie im Durchschnitt bei den Bundestagswahlen von 2005 und 2009 erreicht haben. Die Wahlergebnisse der Bundestagswahlen sind insofern vorteilhaft, da sie die Präferenzen der Wählerinnen und Wähler zum gleichen Zeitpunkt anzeigen.

In der international vergleichenden Literatur ist es durchgehende Praxis, die Effekte parteipolitischer Blöcke und nicht die einzelner Parteien zu analysieren. Dabei wird typischerweise nicht geprüft, ob die in den Blöcken enthaltenen Parteien tatsächlich alle einen gleichgerichteten Effekt auf die jeweilige abhängige Variable haben. Untersucht wird hingegen der Effekt „linker“ und „christlicher“ Parteienblöcke. Überträgt man dies auf die regionale Ebene in der Bundesrepublik, dann gehören die CDU und die CSU zum christlichen Parteienblock. Ihre Stimmanteile werden daher in einer Variablen mit dem Namen UNION zusammengeführt. Der linke Parteienblock setzt sich aus der SPD und der Partei DIE LINKE zusammen. Damit verbleiben die GRÜNEN als links-progressive Partei. Darüber hinaus wird im Anschluss an die funktionalistische Literatur der Verstädterungsgrad als Kontrollvariable berücksichtigt. Dieser wird durch die Bevölkerungsdichte gemessen. Die Zusammensetzung der einzelnen Variablen ist in Tabelle 1 zusammengefasst.

\section{Empirische Analysen}

Die Analyse gliedert sich in zwei Teile: Im ersten Teil wird die Entwicklung der öffentlichen Kleinkinderbetreuung im Zeitverlauf untersucht und der Frage nachgegangen, inwiefern die Betreuungsquoten auf Ebene der Kreise und kreisfreien Städte in West- und Ostdeutschland konvergieren. Der zweite Teil der Untersuchung beschäftigt sich mit den Ursachen der verbleibenden regionalen Varianz und testet die funktionalistischen, parteipolitischen und institutionalistischen Hypothesen. 
TABELLE 1

\section{Zusammensetzung der verwendeten Variablen}

\begin{tabular}{|c|c|c|}
\hline Variable & Kennziffer & Zeitpunkt \\
\hline Kleinkinderbetreuung & Betreuungsquote (Anteil der öffentlich betreuten Kinder unter 3 Jahren an allen unter 3-Jährigen) * & $03 / 2007-03 / 2012$ \\
\hline Wirtschaftlicher Entwicklungsstand & Bruttoinlandsprodukt (BIP) je Einwohner ** & 2009 \\
\hline Frauenerwerbstätigkeit & $\begin{array}{l}\text { Frauenerwerbsquote (Anteil sozialversicherungspflichtig beschäftigter Frauen an weiblicher } \\
\text { Bevölkerung im Alter von } 15 \text { bis unter } 65 \text { Jahren) ** }\end{array}$ & $06 / 2010$ \\
\hline Teilzeittätige Frauen & $\begin{array}{l}\text { Teilzeitquote (Anteil teilzeitbeschäftigter Frauen an allen sozialversicherungspflichtig } \\
\text { beschäftigten Frauen) ** }\end{array}$ & $06 / 2010$ \\
\hline Ehescheidungen & $\begin{array}{l}\text { Scheidungsquote (Anzahl geschiedener Ehen bezogen auf Bevölkerung über } 18 \text { Jahren; } \\
\text { in } 1.000 \text { Personen) }{ }^{* *}\end{array}$ & 2011 \\
\hline Kinderarmut & SGB-II-Quote (Anteil unter 3-Jähriger in Bedarfsgemeinschaften an allen unter 3-Jährigen) **** & $12 / 2011$ \\
\hline UNION & Durchschnittlicher Stimmenanteil von CDU/CSU bei den Bundestagswahlen 2005 und 2009 ** & 2005 bzw. 2009 \\
\hline SPD/LINKE & Durchschnittlicher Stimmenanteil von SPD und DIE LINKE bei den Bundestagswahlen 2005 und $2009 * *$ & 2005 bzw. 2009 \\
\hline GRÜNE & Arithmetisch gemittelter Stimmenanteil der GRÜNEN bei den Bundestagswahlen 2005 und 2009 ** & 2005 bzw. 2009 \\
\hline Bevölkerungsdichte & Einwohner je km²** & $12 / 2010$ \\
\hline
\end{tabular}

Anmerkungen: Bei den drei Kommunalverbänden besonderer Art (Aachen, Hannover, Saarbrücken) wurden geringfügige Anpassungen vorgenommen. Diese und weitere Modifikationen sind im Do-File beschrieben.

Quelle: * Statistische Ämter des Bundes und der Länder 2007-2012; ** „Regionaldatenbank Deutschland“ der Statistischen Ämter des Bundes und der Länder https://www.regionalstatistik.de; *** Statistisches Bundesamt; **** Bundesagentur für Arbeit 2012; Berechnungen der Autoren.

\subsection{Konvergieren die Betreuungsquoten?}

Die auf der linken Seite der in Abbildung 1 wiedergegebene Entwicklung der Betreuungsquoten von Kleinkindern für West- und Ostdeutschland stimmt nur teilweise mit den Thesen von Bonoli (2007) und Fargion (2002) überein. Zunächst einmal ist die ostdeutsche Betreuungsquote sehr viel höher als die im Westen. Hierin zeigt sich erstens, dass die Betreuungsinfrastruktur in den neuen Bundesländern bis heute vom institutionellen Erbe der DDR geprägt ist. Der

\section{ABB. 1}

\section{Entwicklung der Kleinkinderbetreuung 2007-2012}

Angaben in Prozent

-Deutschland gesamt - Westdeutschland - Ostdeutschland

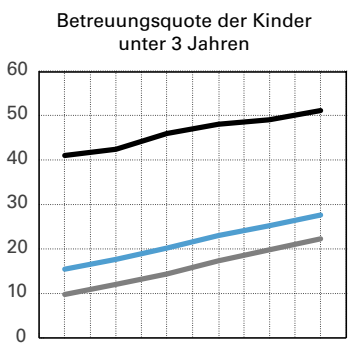

200720082009201020112012
Variationskoeffizient

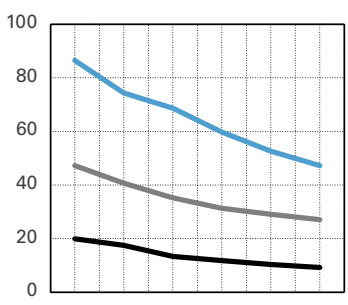

200720082009201020112012
Anmerkungen: West- und Ostdeutschland jeweils ohne Berlin; Variationskoeffizient der Kinderbetreuungsquoten auf Ebene der Kreise und kreisfreien Städte berechnet.

Quelle: Statistische Ämter des Bundes und der Länder

Mitteilungen 2007-2012; Berechnungen der Autoren.
Unterschied zwischen Ost- und Westdeutschland macht einen Großteil der gesamten regionalen Varianz in der Bundesrepublik aus. Die ostdeutsche Betreuungsquote ist zweitens von einem bereits hohen Niveau aus weiterhin angestiegen, was mit der These programmspezifischer Pfadabhängigkeit in Übereinstimmung steht. Allerdings stieg die Betreuungsquote im Westen rascher an als jene im Osten, was wiederum in einem Widerspruch zur crowding out These steht. ${ }^{1}$ Offenbar kann mit dem Aufbau einer Betreuungsinfrastruktur auch nach dem Ende des sogenannten Goldenen Zeitalters des Wohlfahrtsstaates begonnen werden.

Diese Ergebnisse deuten bereits darauf hin, dass es zu einer leichten Konvergenz der Betreuungsquoten in der Bundesrepublik gekommen ist. Auf der rechten Seite von Abbildung 1 ist dazu die zeitliche Entwicklung der Streuung der Betreuungsquoten innerhalb von Westdeutschland, Ostdeutschland und der Bundesrepublik insgesamt abgetragen. Die kontinuierlich sinkenden Variationskoeffizienten belegen, dass das Niveau der öffentlichen Kinderbetreuung zwischen den Kreisen und kreisfreien Städten konvergiert.

\subsection{Determinanten regional variierender Klein- kinderbetreuung in Westdeutschland}

Die obige Diskussion hat verdeutlicht, dass die Betreuungsquoten in Ostdeutschland aufgrund der DDR-Vergangenheit auch heute noch sehr weit über denen im Rest der

1 Im Zeitraum von 2007 bis 2012 stieg die Betreuungsquote in Westdeutschland um 12,5 und in Ostdeutschland um 10,1 Prozentpunkte. 
TABELLE 2

\section{Determinanten der Kleinkinderbetreuung 2012}

\begin{tabular}{lc|c}
\hline AV: Kleinkinderbetreuung & Modell 1 & Modell 2 \\
\hline Wirtschaftlicher Entwicklungsstand & $0,000 * * *$ & $0,000 * *$ \\
Frauenerwerbstätigkeit & $0,315 * *$ & $0,347 * *$ \\
Teilzeittätige Frauen & $0,850 * * *$ & $0,768 * * *$ \\
Ehescheidungen & $1,264 *$ & $1,406 *$ \\
Kinderarmut & $-0,364 * * *$ & $-0,336 * *$ \\
UNION & $-0,519 * * *$ & $-0,243 * * *$ \\
SPD/LINKE & $-0,217 *$ & $0,439 * * *$ \\
GRÜNE & & 0,001 \\
\hline Bevölkerungsdichte & $0,002 *$ & $-20,559 * *$ \\
\hline Konstante & 0,156 & 0,389 \\
$\mathrm{R}^{2}$ & 0,377 & 325 \\
\hline N & 325 & \\
\hline
\end{tabular}

Anmerkungen: Die Fallzahl bezieht sich auf alle 325 Kreise und kreisfreien Städte in Westdeutschland (ohne Berlin) zum Gebietsstand 03/2012; lineare Regressionsmodelle mit robusten Standardfehlern geschätzt; Signifikanzniveaus:

* $\mathrm{p}<0,05 ; * * \mathrm{p}<0,01 ; * * \mathrm{p}<0,001$

Quelle: Statistische Ämter des Bundes und der Länder 2007-2012; „Regionaldatenbank Deutschland der Statistischen Ämter des Bundes und der Länder, https://www.regionalstatistik.de;

Statistisches Bundesamt; Bundesagentur für Arbeit 2012; Berechnungen der Autoren.

Republik liegen. Die Bundeshauptstadt Berlin und die Regionen der neuen Länder werden daher aus den folgenden Querschnittsanalysen ausgeklammert. ${ }^{2}$ Die Analysen beziehen sich also nur auf die 325 westdeutschen Kreise und kreisfreien Städte. Inwiefern können nun die operationalisierten Variablen die Unterschiede im Ausbau der Kleinkinderbetreuung erklären? Tabelle 2 zeigt dazu die Ergebnisse zweier multivariater Querschnittsregressionen.

Der Einfluss des wirtschaftlichen Entwicklungsstandes auf den Ausbau der Kleinkinderbetreuung weist, wie erwartet, einen positiven Zusammenhang auf und ist in beiden Modellen hoch signifikant.

Auch die zunehmende Arbeitsmarktintegration von Frauen steht in beiden Modellen in einem positiven und hoch signifikanten Zusammenhang mit dem Anteil der öffentlich betreuten Kleinkinder. Der ebenfalls positive Koeffizient der Teilzeitquote deutet zudem darauf hin, dass die Differenz zwischen den Regionen insbesondere durch den Grad der weiblichen Teilzeitbeschäftigung erklärt werden kann. Die beiden Beschäftigungsvariablen bestätigen die Ergebnisse vorangegangener Studien (Bonoli/Reber 2010, S. 113; Huber/ Stephens 2000, S. 331ff.). Allerdings muss berücksichtigt werden, dass nicht nur die Erwerbsquoten auf die Kinderbetreuung wirken, sondern auch umgekehrt der Ausbau der Kinderbetreuung die weibliche Beschäftigung begünstigen kann. Um einem möglichen Kausalitätsproblem zu begegnen, haben wir mit älteren Beschäftigungsdaten aus dem Jahre 2008 experimentiert. Diese im Online-Appendix (Tabelle III) dokumentierte Änderung am Modell hat jedoch keinen bedeutsamen Effekt. Das Signifikanzniveau der Frauenerwerbstätigkeit verringert sich nur geringfügig.
Die mittels Scheidungsraten erfassten neuen Familienstrukturen haben ebenfalls einen positiven und signifikanten Effekt auf die regional variierende Kleinkinderbetreuung. Dies kann dahingehend interpretiert werden, dass Alleinerziehende eine stärkere Nachfrage nach Kinderbetreuungsplätzen entfalten, was die Kommunen angesichts der im August 2013 in Kraft tretenden Betreuungsgarantie wiederum dazu veranlassen könnte, die öffentliche Kinderbetreuung auszudehnen, um so Klagen seitens der Elternteile zu vermeiden. Grafische Analysen dieser Variablen zeigen jedoch, dass hier Ausreißer eine vergleichsweise große Rolle spielen. Zudem erwies sich die Scheidungsquote als am wenigsten robuste Variable in den Modellen.

Erstaunlich ist der in beiden Modellen negative und höchst signifikante Zusammenhang zwischen Kinderarmut und dem Anteil der öffentlich betreuten Kinder. Je mehr Kinder unter drei Jahren auf Leistungen nach dem SGB II angewiesen sind, desto geringer ist der Anteil der öffentlich betreuten Kleinkinder in der entsprechenden Region. Die Höhe der Betreuungsquote richtet sich also nicht nach dem funktionalen Erfordernis der Armutsvermeidung. Dies deutet darauf hin, dass Eltern im SGB-II-Bezug ihre Kinder häufiger selbst betreuen (müssen) und daher eine geringere Nachfrage nach Kinderbetreuungsplätzen entfalten. Zugleich gehen Arbeitslose weitaus seltener zur Wahl als der Durchschnitt der Bevölkerung (Schäfer 2012, S. 247ff.). Die Gruppe der Eltern, die Leistungen nach dem SGB II bezieht, fragt also weniger öffentliche Betreuung nach und übt weniger Druck auf ein erweitertes Angebot aus. Die umgekehrte Interpretation, wonach die Kinderarmut besonders niedrig ist, wo ein ausreichendes Betreuungsangebot es Eltern ermöglicht, den SGB-II-Bezug ihrer Kinder durch Erwerbstätigkeit zu vermeiden, erscheint spätestens seit den Leistungsverbesserungen beim Kinderzuschlag im Jahre 2008 unplausibel.

Damit kommen wir schließlich zu den politischen Variablen, welche den Unterschied beider Modelle begründen. Modell 1 umfasst - wie in der vergleichenden Wohlfahrtsstaatsforschung üblich - die Stimmenanteile der christlichen (UNION) und die der linken Parteien (SPD/LINKE). Das zweite Modell geht hingegen von einem Modell des Parteienwettbewerbs aus, bei dem der Konflikt um die Kinderbetreuung zwischen links-progressiven (GRÜNE) und rechtskonservativen (UNION) Kräften ausgefochten wird.

Es zeigt sich, dass die christlichen Parteien im ersten Modell einen Effekt aufweisen, der mit den theoretischen Erwartungen übereinstimmt. Je größer der Stimmenanteil der Unionsparteien, desto geringer ist der Anteil der betreuten Kleinkinder in den westdeutschen Kreisen. Es über-

2 Separate Analysen für Ostdeutschland sind im Online-Appendix (Tabelle Il; http://www.boeckler.de/pdf/wsi_seils_ meyer_online_appendix_2013.pdf) einsehbar. Die Koeffizienten in diesen Modellen sind allerdings weitgehend insignifikant, weil das institutionelle Erbe der DDR stark dominiert. 
rascht, dass der Effekt so deutlich ausfällt, da dies auf eine erhebliche Kluft zwischen der Wählerschaft von CDU/CSU und der von ihr forcierten Bundespolitik hindeuten würde. Betrachtet man nun den signifikanten Koeffizienten von SPD/LINKE, kommen Zweifel daran auf, ob dieses Modell den Konflikt um die Kinderbetreuung adäquat abzubilden vermag. Je höher die Stimmenanteile linker Parteien in westdeutschen Kreisen, desto geringer sind im Durchschnitt die Betreuungsquoten bei den Kleinkindern.

Weitere Analysen, die im Online-Appendix (Tabelle IV) dokumentiert sind, zeigen zudem, dass die Stimmenanteile der SPD und der Partei DIE LINKE in unterschiedlicher Weise mit dem Ausmaß der Kleinkinderbetreuung in den Kreisen zusammenhängen. Während von der SPD ein höchst signifikanter negativer „Effekt“ ausgeht, ist dieser bei der Partei DIE LINKE signifikant positiv. Dieser Befund führt also zu zwei Schlussfolgerungen: Erstens ist die in der Literatur getroffene Annahme eines homogenen, linken Parteienblocks so nicht zutreffend. Zweitens zeigt sich im Gegensatz zur Literatur (Huber/Stephens 2000; Iversen/Stephens 2008) kein durchweg positiver „Effekt“ linker Parteien auf die Betreuung von Kleinkindern.

Welche Ergebnisse liefert das Alternativmodell? Erstens reduziert sich der hoch signifikante Koeffizient des Stimmenanteils der christlichen Parteien um die Hälfte. Dies kann im Modell von Kitschelt dahingehend interpretiert werden, dass die Führung der Unionsparteien neue Wählerschichten erobern will und deshalb den Ausbau der Kleinkinderbetreuung vorantreibt, welchem ihre traditionelle Klientel kritisch gegenübersteht. Zweitens zeigt sich, dass der Stimmenanteil der links-progressiven GRÜNEN, deren Klientel sich sehr für öffentliche Kinderbetreuung interessiert, positiv und höchst signifikant mit dem Umfang der Kleinkinderbetreuung zusammenhängt.

\section{Fazit}

In diesem Beitrag wurde der Versuch unternommen, die klassischen Theorien der international vergleichenden Staatstätigkeitsforschung auf regionale Unterschiede bei der öffentlichen Betreuung von Kindern unter drei Jahren anzuwenden. In einem ersten Schritt wurde dargelegt, wie die alten „industriellen“ Theorien des Funktionalismus, der Parteiendifferenztheorie und des Institutionalismus genutzt werden können, um Hypothesen zur Erklärung von Unterschieden im „post-industriellen“ Wohlfahrtsstaat zu gewinnen. Diese dienten der Hypothesenbildung, um die regional erheblich variierende Betreuungssituation für Kleinkinder im Bundesgebiet zu erklären.

Die Bedeutung des institutionellen Erbes konnte anhand eines OstWest-Vergleichs demonstriert werden. Einerseits zeigte sich, dass die $\mathrm{Ni}$ veauunterschiede zwischen den beiden Regionen praktisch zur Gänze auf die Nachwirkungen der Kinderbetreuungspolitik in der DDR zurückzuführen sind. Andererseits konnte im Gegensatz zu populären institutionalistischen Hypothesen kein Einfluss des institutionellen Erbes auf die Entwicklung der Betreuungsquoten der Kinder unter drei Jahren festgestellt werden. Vielmehr steigen die Betreuungsquoten im Westen schneller als im Osten, sodass es zu einer Konvergenz der Betreuungsquoten im Bundesgebiet kommt.

Obschon funktionalistische Variablen in der neueren Wohlfahrtsstaatsforschung in Ungnade gefallen sind, liefern sie einen bedeutenden Beitrag zur Erklärung der Varianz der Betreuungsquoten zwischen den westdeut- schen Kreisen. Je höher der wirtschaftliche Entwicklungsstand, je ausgedehnter die Frauenerwerbstätigkeit und je weiter die Auflösung von Ehe und Familien vorangeschritten sind, desto höher ist im Durchschnitt die Betreuungsquote der Kleinkinder in einem Kreis. Die Verbreitung von Kinderarmut hat hingegen einen Effekt, der funktionalistischen Erwartungen zuwider läuft: Je mehr Kinder auf Leistungen nach dem SGB II angewiesen sind, desto geringer ist die Betreuungsquote unter Kleinkindern.

Mit Bezug auf die parteipolitischen Hypothesen zeigte sich, dass das traditionelle Rechts-Links-Schema zur Erklärung der Kinderbetreuung wenig geeignet erscheint. Der Stimmanteil traditionell linker Parteien hängt - im Gegensatz zu den Ergebnissen in der Literatur (Bonoli/Reber 2010, S. 113ff.; Huber/Stephens 2000, S. 332) - nicht durchweg positiv mit dem Umfang der Kleinkinderbetreuung auf der Kreisebene zusammen. Außerdem kann die Annahme eines homogenen linken Parteienblocks, wie er in der Literatur vorherrscht, nicht bestätigt werden. Geeignet ist hingegen der Analyserahmen von Herbert Kitschelt, der die Kinderbetreuung in einem Spannungsfeld zwischen rechts-konservativen und links-progressiven Parteien verortet. Es zeigt sich, dass vor allem ein hoher Stimmenanteil der links-progressiven GRÜNEN mit einer ausgedehnten Betreuungsinfrastruktur verbunden ist. Kreise, in denen die Christdemokraten bei Bundestagswahlen erfolgreich sind, haben hingegen im Durchschnitt eine niedrigere Betreuungsquote in dieser Altersgruppe. Dies zeigt, dass der von den Christdemokraten auf der Bundesebene forcierte Ausbau der Kinderbetreuung im Gegensatz zu dem steht, was ihre Partei bzw. Klientel auf der Kreisebene bislang wünscht. Wenngleich sich die Präferenzen der Wählerschaft der UNION unter dem Eindruck des Wertewandels ändern mögen, kann dieses Ergebnis dahingehend interpretiert werden, dass sich die Parteiführung neuen Wählerschichten öffnen oder Koalitionen mit den GRÜ NEN ermöglichen will.

\section{LITERATUR}

Achinger, H. (1979 [1958]): Sozialpolitik als Gesellschaftspolitik. Von der Arbeiterfrage zum Wohlfahrtsstaat, Frankfurt a. M.

Bonoli, G. (2007): Time Matters: Postindustrialization, New Social Risks, and Welfare State Adaptation in Advanced Industrial Democracies, in: Comparative Political Studies 40 (5), S. 495-520

Bonoli, G./Palier, B. (2007): When Past Reforms Open New Opportunities: Comparing Old-age Insurance Reforms in Bismarckian Welfare Systems, in: Social Policy \& Administration 41 (6), S. 555-573

Bonoli, G./Reber, F. (2010): The Political Economy of Childcare in OECD Countries: Explaining Cross-national Variation in Spending and Coverage Rates, in: European Journal of Political Research 49 (1), S. 97-118

Bundesagentur für Arbeit (2012): Kinder in Bedarfsgemeinschaften (Dezember 2011) Nürnberg

Ellingsæter, A. L. (2008): The Provision of Childcare Services in Norway. External Report Commissioned by and Presented to the EU Directorate-General Employment and Social Affairs, Unit G1 "Equality between Women and Men", Manuskript

Emerek, R. (2008): The Provision of Childcare Services in Denmark. External Report Commissioned by and Presented to the EU Directorate-General Employment and Social Affairs, Unit G1 „Equality between Women and Men”, Manuskript

Esping-Andersen, G. (1996): After the Golden Age? Welfare State Dilemmas in a Global Economy, in: Esping-Andersen, G. (Hrsg.): Welfare States in Transition: National Adaptations in Global Economies, London, S. 1-31

Esping-Andersen, G. (1999): Social Foundations of Postindustrial Economies Oxford

Esping-Andersen, G. (2002): A Child-centred Social Investment Strategy, in: Esping-Andersen, G./Gallie, D./Hemerijck, A./Myles, J. (Hrsg.): Why We Need a New Welfare State, Oxford, S. 26-67 
Fargion, V. (2002): Timing and the Development of Social Care Services in Europe, in: Ferrera, M./Rhodes, M. (Hrsg.): Recasting European Welfare States,

London S. 59-88

Henninger, A./von Wahl, A. (2010): Das Umspielen von Veto-Spielern: Wie eine konservative Familienministerin den Familialismus des deutschen Wohlfahrts staates unterminiert, in: Egle, C./Zohlnhöfer, R. (Hrsg.): Die zweite Große Koalition: Eine Bilanz der Regierung Merkel (2005-2009), Wiesbaden, S. 361-379 Hibbs, D. A. (1977): Political Parties and Macroeconomic Policy, in: American Political Science Review 71 (4), S. 1467-1487

Huber, E./Ragin, C./Stephens, J. D. (1993): Social Democracy, Christian Democracy, Constitutional Structure, and the Welfare State, in: American Journa of Sociology 59 (3), S. $711-749$

Huber, E./Stephens, J. D. (2000): Partisan Governance, Women's Employment and the Social Democratic Service State, in: American Sociological Review 65 (3), S. 323-342

Iversen, T./Stephens, J. D. (2008): Partisan Politics, the Welfare State, and Three Worlds of Human Capital Formation, in: Comparative Political Studies $41(4 / 5)$, S. $600-637$

Jaumotte, F. (2003): Labour Force Participation of Women: Empirical Evidence on the Role of Policy and other Determinants in OECD Countries, in: OECD Economic Studies 37 (2), S. 51-108

Kitschelt, H. (1993): Class Structure and Social Democratic Party Strategy, in: British Journal of Political Science 23 (3), S. 299-337

Kitschelt, H. (1999): European Social Democracy between Political Economy and Electoral Competition, in: Kitschelt, H./Lange, P./Marks, G./Stephens, J. D (Hrsg.): Continuity and Change in Contemporary Capitalism, Cambridge,

S. 317-345

Kitschelt, H./Rehm, P. (2006): New Social Risk and Political Preferences, in: Armingeon, K./Bonoli, G. (Hrsg.): The Politics of Post-Industrial Welfare States: Adapting Post-war Social Policies to New Social Risks, London, S. 52-82 Organisation for Economic Co-operation and Development (OECD) (2011): Doing Better for Families, Paris

Orloff, A. S./Skocpol, T. (1984): Why not Equal Protection? Explaining the Politics of Public Social Spending in Britain, 1900-1911, and the United States, 1880-1920, in: American Sociological Review 49 (6), S. 726-750

Pierson, P. (1994): Dismantling the Welfare State? Reagan, Thatcher, and the Politics of Retrenchment, Cambridge

Pierson, P. (1996): The New Politics of the Welfare State, in: World Politics 48 (2), S. $143-179$

Pierson, P. (2000): Increasing Returns, Path Dependence, and the Study of Politics, in: American Political Science Review 94 (2), S. 251-267

Pierson, P./Skocpol, T. (2000): Historical Institutionalism in Contemporary Political Science: American Political Science Association Meetings, Washington
Randall, V. (2000): Childcare Policy in the European States: Limits to Convergence, in: Journal of European Public Policy 7 (3), S. 346-368

Schäfer, A. (2012): Beeinflusst die sinkende Wahlbeteiligung das Wahlergebnis? Eine Analyse kleinräumiger Wahldaten in deutschen Großstädten, in: Politische Vierteljahresschrift 53 (2), S. 240-264

Statistische Ämter des Bundes und der Länder (2007-2012): Kindertagesbetreuung Regional 2007-2012, Wiesbaden

Statistische Ämter des Bundes und der Länder (2012): Kindertagesbetreuung Regional 2012, Wiesbaden

Statistisches Bundesamt (2012a): 27,6 \% der unter 3-Jährigen zum 1. März 2012 in Kindertagesbetreuung - 220000 Plätze fehlen noch, Pressemitteilung vom 06.11., Wiesbaden

Statistisches Bundesamt (2012b): Kindertagesbetreuung in Deutschland 2012 Wiesbaden

Whiteford, P./Adema, W. (2007): What Works Best in Reducing Child Poverty: A Benefit or Work Strategy?, Paris

Wilensky, H. L. (1975): The Welfare State and Equality: Structural and Ideological Roots of Public Expenditures, Berkeley

Wilensky, H. L. (1981): Leftism, Catholicism, and Democratic Corporatism:The Role of Political Parties in Recent Welfare State Development, in: Flora, P./Heidenheimer, A. J. (Hrsg.): The Development of Welfare States in Europe and America, New Brunswick, S. 345-382

Zöllner, D. (1963): Öffentliche Sozialleistungen und wirtschaftliche Entwicklung: Ein zeitlicher und internationaler Vergleich, Berlin

\section{AUTOREN}

ERIC SEILS, Dr., ist Wissenschaftler im Wirtschafts- und Sozialwissenschaftlichen Institut (WSI) in der Hans-Böckler-Stiftung. Arbeitsschwerpunkte: Vergleichende Wohlfahrtsstaatsforschung, Armut, Politische Ökonomie.

eric-seils@boeckler.de

DANIEL MEYER studiert Sozialökonomik und Soziologie an der Universität Erlangen-Nürnberg.

meyer_daniel@arcor.de 